\title{
CRÉDITO AL CONSUMO EN ARGENTINA DURANTE PERIODOS NORMALES Y DE CRISIS ECONÓMICAS
}

\author{
Juliana E. Vega Lacorte \\ Karen Watkins Fassler*
}

(Recibido: Noviembre 2012 / Aprobado: Abril 2013)

\section{Resumen}

Esta investigación indaga sobre la relación entre factores macroeconómicos y la decisión de las familias para financiar su consumo con deuda. Particularmente, se estudia la correspondencia entre el ingreso, la tasa de interés, el desempleo y la confianza de los consumidores con el endeudamiento de las familias. Se verifica también si las relaciones entre las variables macroeconómicas (particularmente el ingreso) y la deuda para consumo son coherentes con las teorías que se han escrito sobre el consumo. Como estudio de caso se toma Argentina, pues permite el análisis bajo distintos escenarios normales y de crisis económicas (1994-2011).

Palabras clave: crédito al consumo, crisis económicas, Argentina Clasificación JEL: E21

\section{Abstract}

This paper deals with the relationship between macroeconomic factors and consumption debt. Specifically, it studies the association between income, interest rate, unemployment, and consumer confidence with consumption debt. The relationship between macroeconomic variables (in particular

* Posgrado y Centro de Investigación e Inteligencia Económica, Universidad Popular Autónoma del Estado Puebla. Correo electrónico: <juliana.vlacorte@gmail.com> y <karen.watkins@upaep.mx>. 
Juliana E. Vega / Karen Watkins

income) and consumption debt is verified according to diverse consumption theories. Argentina is taken as case study, as it allows performing the analysis under diverse scenarios of normal and crisis episodes (1994-2011).

Keywords: consumption debt, economic crises, Argentina JEL Classification: E21

\section{Introducción}

Organismos tales como la Comisión Europea y el Bank for International Settlements (BIS) señalan que en años recientes el endeudamiento para consumo de las familias ha experimentado un notable crecimiento en varias regiones del mundo. América Latina no es la excepción; sin embargo, la escasa investigación al respecto en esa parte del globo limita el conocimiento del comportamiento y determinantes de este mercado crediticio y sus efectos sobre el dinamismo económico.

Esta investigación busca conocer el impacto de algunos factores macroeconómicos sobre la decisión de las familias para financiar su consumo con deuda. Particularmente, se estudia la relación entre el ingreso, la tasa de interés, el desempleo y la confianza de los consumidores con el endeudamiento de las familias. Como estudio de caso se toma Argentina, pues permite el análisis bajo distintos escenarios normales y de crisis económica. Tal como lo menciona Lo Vuolo en el 2003 (haciendo referencia a Argentina): “Es poco común encontrar países que en una década se muevan de una hiperinflación a una depresión económica, separados ambos episodios por un periodo de aparente bonanza y un generalizado clima de 'satisfacción' por gran parte de la sociedad". Además, el consumo privado representa en Argentina el 80\% del PIB, siendo uno de los principales componentes de la producción.

Se busca también con este trabajo verificar si las relaciones entre las variables macroeconómicas (particularmente el ingreso) y la deuda para consumo en Argentina son coherentes con una "racionalidad económica" desde el punto de vista de las teorías que se han escrito sobre el consumo. Específicamente, se hace referencia a las hipótesis planteadas por Keynes, Duesenberry, Friedman y Modigliani.

El trabajo está organizado de la siguiente manera: en la segunda sección se hace referencia a las teorías de consumo. En el tercer apartado se expone la historia y teoría respecto al crédito para consumo. En el apartado 
cuarto se especifican datos y variables utilizados en esta investigación. En la quinta sección se identifican los episodios de crisis económicas en Argentina. La sección sexta incluye el análisis descriptivo del estudio. El apartado séptimo se refiere al análisis econométrico. Finalmente, la octava sección concluye esta investigación.

\section{Teorías de consumo}

\subsection{La hipótesis del ingreso absoluto}

Esta hipótesis se plantea inicialmente en La Teoría general de la ocupación, el interés y el dinero (Keynes, 1936) y se expande posteriormente. De acuerdo con Keynes, cuando el ingreso corriente aumenta, el gasto en consumo también se eleva aunque no necesariamente en la misma proporción. A partir de esta relación de corto plazo, se construye una curva que muestra la cantidad de consumo asociada a distintos niveles de ingreso, la cual llama Keynes propensión marginal a consumir. Es así que las variaciones en la cantidad de consumo dependen principalmente de las variaciones de la cantidad absoluta de ingreso y no de cambios en la propensión con un ingreso dado.

\subsection{La hipótesis del ingreso relativo}

Otra de las grandes contribuciones a la teoría del consumo surgió cuando James Duesenberry se preocupó por considerar la dimensión social que afecta el consumo de las familias. En su libro: Income, Saving and the Theory of Consumer Behavior (1949), Duesenberry criticó la función de consumo keynesiana y reformuló la teoría del consumo (Mason, 2000). Esencialmente, la falla en la hipótesis del ingreso absoluto de Keynes, según Duesenberry, fue dejar en el olvido la influencia que tiene el contexto social y psicológico en las decisiones de consumo y ahorro de las familias.

Incorporar la dimensión social en el análisis de una función de consumo significó aclarar que el comportamiento de consumo de un individuo está definido no sólo por sus preferencias personales en aislamiento sino por sus preferencias personales en interacción con las decisiones de consumo del resto de la población. Es decir, que los comportamientos de consumo son interdependientes y por tanto a las personas les preocupa más su nivel de consumo en comparación con el consumo que realicen los demás. 
Juliana E. Vega / Karen Watkins

Con funciones de utilidad interdependientes, como lo postuló Duesenberry, el consumo de los individuos se encuentra -hasta cierto punto- determinado socialmente. Y esta interdependencia implica que las decisiones de gasto en consumo involucren un componente relacionado con el hábito personal y con la imitación de hábitos de sectores de ingresos más altos (Palley, 2007). Los patrones de consumo son difíciles de ajustar a la baja cuando ocurren reducciones en el ingreso. El consumo es entonces una función del ingreso relativo, tanto del ingreso o consumo de los otros como del ingreso o consumo pasados (Jiménez, 2001).

\subsection{La hipótesis del ingreso permanente y del ciclo vital}

En el libro: A Theory of Consumption Function (1957), Milton Friedman dio forma a una nueva teoría conocida como "hipótesis del ingreso permanente". En ésta relacionó la función de consumo con un ingreso a lo largo de la vida en lugar de un ingreso corriente. La finalidad de la hipótesis del ingreso permanente es explicar la tendencia que tienen los individuos a ajustar el gasto en consumo, con el tiempo, a su corriente de ingreso medio (Makin, 1977). Los individuos siguen esta tendencia porque desean mantener una senda de consumo estable a lo largo de su vida.

Similar a lo que Milton Friedman hizo con su "hipótesis del ingreso permanente", Franco Modigliani desarrolló su propia teoría del consumo, junto con su estudiante Richard Brumberg, partiendo de un análisis orientado hacia el futuro. La diferencia radica en que estos autores fundamentaron su teoría en la idea de que las personas toman decisiones inteligentes acerca de cuánto desean gastar en cada etapa de su vida (juventud, edad media, vejez) siendo su única restricción los recursos disponibles a lo largo de la vida (Deaton, 2005). Así, las personas ahorran durante sus años de trabajo con la finalidad de mantener un patrón de consumo no tan distinto en los años de jubilación.

\section{El crédito al consumo}

\subsection{Historia}

A lo largo del siglo xIx, adquirir con crédito cualquier bien de lujo o no esencial era visto como imprudente y hasta inmoral. Esta práctica de financiar 
consumo "lujoso" estaba reservada únicamente para los adinerados, quienes compraban muebles costosos, objetos de cristal y elegantes ropas. Era usual también que los granjeros pidieran crédito para comprar semillas ya que pagarían después en el tiempo de cosecha (Boorstin, 2004).

Por otra parte, el crédito se daba prácticamente sólo entre conocidos, lo cual reducía los problemas de información asimétrica pero restringía el acceso al financiamiento. A fines del siglo xIX, las únicas opciones para financiar el consumo consistían en pedir prestado a familiares, a usureros o acudir a las casas de empeño.

En 1920 se expandió el financiamiento al consumo en Estados Unidos de Norteamérica. La gran producción de bienes durables motivó a las tiendas a buscar medios para impulsar la compra de estos bienes. La manera en que los nuevos productos estuvieran al alcance de todos era expandir el uso del crédito a plazos. El primer impulso lo facilitaron las compañías automotrices al ofrecer a sus clientes la opción de un pago inicial y cuotas mensuales. Así, hasta los inicios de los años cincuenta, los que ofrecían el crédito a las familias eran los mismos que les vendían los productos. Pero entonces surgieron nuevas propuestas como las tarjetas de crédito de Diners Club, y de ahí las primeras tarjetas bancarias como Visa y MasterCard (Boorstin, 2004). Estos nuevos instrumentos tuvieron éxito gracias a que en 1952 se terminaron las restricciones al crédito impuestas durante el periodo de guerra, surgió el baby boom, se insertaron las mujeres a la fuerza de trabajo y las familias se mostraron más dispuestas a utilizar el crédito para su consumo (Calder, 2002). Para 1960, la innovación financiera y la nueva actitud de los consumidores hacia el crédito, permitió el despegue de un uso generalizado y habitual de financiar consumo con planes a cuotas y tarjetas de crédito.

\subsection{Revisión de la literatura sobre el crédito al consumo}

En su libro: The Impact of Public Policy on Consumer Credit, Durkin y Staten (2002) elaboran un capítulo acerca del desarrollo que ha tenidola investigación en el campo de las finanzas del consumidor -concretamente en el tema del crédito al consumo. Mencionan estos autores que unos de los primeros estudios en esta área los realizó a partir de 1940 el National Bureau of Economic Research (NBER). Estas investigaciones respondían al interés del gobierno de los Estados Unidos de Norteamérica por comprender las repercusiones macroeconómicas del crédito al consumo. Los títulos se enfocaron a temas como el crédito y el ciclo de negocios, la regulación y el control de la oferta 
Juliana E. Vega / Karen Watkins

de crédito. En esta línea de investigación se ubican trabajos iniciales como el de Haberler (1942), quien concluye que el crédito al consumo genera efectos adversos sobre la estabilidad económica, pues acentúa los periodos de prosperidad económica a la vez que intensifica los periodos de depresión. Contrario a este planteamiento, Cox (1948) establece que las fluctuaciones en el nivel agregado del crédito a plazos debieran reflejar los cambios del estado general del entorno económico y no precisamente desencadenarlos.

A medida que los expertos profundizaban en el tema, surgieron trabajos que planteaban los factores detrás de la demanda de créditos al consumo. Debido a la falta de una teoría específica y directa que abordara el uso del crédito para comprar bienes, la mayoría de los autores se apoyaron de una $\mathrm{u}$ otra forma en las teorías del consumo desarrolladas por Duesenberry y Modigliani. Es así que en varios de estos trabajos se vislumbró el crédito al consumo como función esencialmente del ingreso o de la distribución del ingreso (Kisselgoff, 1952 y Dauten, 1954). Hubo también quienes mencionaron como factores explicativos del crédito al consumo el número de matrimonios (que lleva a un mayor consumo de bienes durables), las tasas de interés, los montos de los pagos mensuales del crédito y su plazo (Kisselgoff, 1952).

Además de enriquecer la investigación con el análisis acerca de las causas que determinan la demanda de créditos al consumo, los estudios al respecto también se preocuparon por el rápido crecimiento que estaban experimentando a partir de 1955. Se argumentaba que el número de familias crecía a tasas cada vez más altas y que los consumidores estaban más dispuestos a adquirir deuda porque deseaban mejorar su estándar de vida y a la vez se sentían más seguros de poder pagar la deuda en el futuro (Lewis, 1956 y Enthoven, 1957).

A partir de los años sesenta, el sector privado se interesó cada vez más en los estudios del crédito al consumo, con lo que se dieron subvenciones al NBER para que continuara elaborando los mismos. Aunque estos trabajos siguieron prestando atención a cuestiones macro, su énfasis fue en temas micro como la calidad del crédito, la sensibilidad hacia las tasas de interés, la incertidumbre respecto del ingreso y la estructura de costos financieros, entre otros (Pearce, 1985). Ya para finales del siglo xx, los temas recurrentes en las investigaciones del NBER tenían que ver con el crecimiento del crédito, la preocupación por la carga de la deuda, la innovación tecnológica y el acceso al crédito. En esta época la explicación al crecimiento de la deuda de las familias para financiar consumo se buscó en razones históricas (relacionadas al ciclo económico y los cambios demográficos) y en los cambios regulatorios 
que sufría el mercado de créditos, particularmente a partir de la desregulación financiera y las innovaciones que surgieron a raíz de esta liberalización.

A partir del año 2000, particularmente entre 2001 y 2006 (previo a la crisis financiera mundial), los niveles de endeudamiento para el consumo alcanzaron niveles récord. La explicación para ello se encuentra en varias investigaciones (Barnes y Young, 2003; Weller, 2007), donde continúan siendo significativos los efectos sobre el crédito de las tasas de interés, las expectativas de crecimiento del ingreso (dada una falta de ingreso corriente) y los cambios demográficos.

\subsection{Factores explicativos del crédito al consumo}

\subsubsection{El ingreso}

Una de las variables que encabeza la lista de factores determinantes en la decisión de pedir prestado es el ingreso de las familias. A pesar de que diversos autores coinciden en que el ingreso tiene un efecto importante sobre la demanda de créditos de consumo, discrepan en cuanto a la dirección que explica tal relación, ya que han encontrado en los análisis de regresión coeficientes tanto positivos como negativos. Por ejemplo, Chrystal y Mizen (2001), Del Río (2002) y, Chen Chen y Chivakul (2008) concluyen que existe una fuerte relación positiva entre la cantidad de deuda que poseen los hogares y su ingreso; a medida que aumenta el ingreso se incrementa también la capacidad de endeudamiento y el gasto de los consumidores. Se vuelve psicológicamente más fácil endeudarse ya que aumenta la confianza respecto al ingreso futuro (Strebkov, 2005). Además, por el lado de la oferta, los límites del crédito se expanden cuando se incrementa el nivel de ingreso real (Ashley, 2002). Por otro lado, autores como Magri (2002) señalan que cuando el ingreso aumenta las familias son capaces de financiar una mayor parte del consumo con recursos propios, por lo que debería caer la demanda por préstamos. Es más, para niveles de ingreso bajos, la utilidad marginal del consumo es muy alta lo cual se traduce en una alta demanda por préstamos (Chow, 1960).

\subsubsection{La tasa de interés}

El precio de los préstamos al consumo es la tasa de interés real que cobran los bancos a sus clientes por el uso de estos créditos. Es lógico esperar que las tasas de interés tengan un efecto sobre las decisiones de pedir prestado 
ya que indican cuánto del ingreso futuro estará comprometido para el pago de intereses. Entre más elevadas sean las tasas de interés de los créditos de consumo, más grande será el sacrificio del ingreso futuro para alcanzar un mismo nivel de consumo presente financiado con deuda (Park, 1993). En consecuencia, cuando se observan niveles bajos en las tasas de interés, endeudarse se transforma en una opción más atractiva que antes (Barnes y Young, 2003; Tudela y Young, 2005; Morales y Yáñez, 2006).

Aunque no hay duda de que la tasa de interés se relaciona negativamente con el nivel de deuda, sí existe controversia alrededor de la significancia que tiene ésta sobre la decisión de endeudamiento. Los resultados para Gross y Souleles (2001) indican que la tasa de interés sí es significativa, contrario a los hallazgos de autores tales como Ashley (2002) y Del Río (2002). La poca reacción de los consumidores ante movimientos en las tasas de interés se puede atribuir a los costos de información, la falta de conocimiento crediticio o el hecho de que existen consumidores que pagan sus tarjetas de crédito a tiempo y por ende no incurren en pagos de intereses (Chen, 2007).

\subsubsection{El desempleo}

En cuanto al comportamiento del desempleo como variable determinante del endeudamiento, una primera aproximación es que la relación vaya en sentido positivo. Se espera que los desempleados estén más dispuestos a adquirir deuda debido a que no desean comprometer su consumo actual por falta de ingreso corriente, siempre que el desempleo sea temporal (Chow, 1960). No obstante, por el lado de la oferta los prestamistas son reacios a otorgar créditos cuando no se garantiza una fuente segura de ingresos, por lo que los desempleados son más propensos a experimentar restricciones crediticias (Chen Chen y Chivakul, 2008).

Se ha observado que el crecimiento en el otorgamiento de créditos al consumo se origina durante tiempos de poco desempleo (Logemann, 2008). De acuerdo a autores tales como Del Río (2002), Soto (2004) y, Neagu y Margarit (2007), bajo estas circunstancias el ingreso esperado aumenta, se reduce la incertidumbre sobre este ingreso y se contraen las restricciones al crédito.

\subsubsection{La confianza del consumidor}

Las expectativas de las familias, tanto de su situación financiera personal como de la economía en general, tienden a reflejar niveles de incertidumbre 
sobre el ingreso futuro. El indicador de confianza del consumidor integra esas expectativas y hace posible crear una medición de incertidumbre que, de acuerdo con Dion (2006), se necesita para poder estudiar mejor la dinámica de corto plazo del consumo. Como lo subraya Baumohl (2007), las familias se encuentran más a gusto adquiriendo deuda y consumiendo cuando se sienten seguras de su trabajo e ingresos futuros.

Ante la incertidumbre, la pregunta ¿qué tanto pueden consumir las familias? cede el paso a una más importante, ¿qué tanto están dispuestas a consumir? La capacidad de consumir está definida por el ingreso disponible (Earl y Kemp, 2002). Pero las variables económicas no siempre capturan cuál será el ingreso disponible futuro con el que las familias forman sus decisiones de endeudamiento y consumo. Entonces, la disposición a consumir no puede ser explicada únicamente por la reacción de los consumidores ante variables económicas actuales (Desroches y Gosselin, 2002).

Bajo esta óptica, es válido suponer que exista una relación positiva entre la confianza de los consumidores y la cantidad de préstamos que toman para el consumo. Por ejemplo, Wilcox (2007) sostiene que la confianza del consumidor es un útil complemento de las variables ingreso y tasa de interés. Sin embargo, otros autores han expresado su preocupación en torno a la utilización del índice de confianza en los modelos econométricos. De acuerdo a Desroches y Gosselin (2002), y Ludvigson (2004), la confianza del consumidor podría reflejar los fundamentos económicos actuales en vez de capturar factores psicológicos exógenos a los primeros.

\section{Datos y variables}

Los datos se obtuvieron de tres fuentes: Banco Central de la República Argentina (BCRA), Instituto Nacional de Estadística y Censos (INDEC) y Centro de Investigación en Finanzas de la Universidad Torcuato di Tella (CIF). Las variables cubren mensualmente el periodo enero 1994 a junio 2011, con excepción del Índice de Confianza del Consumidor (ICC), que inicia en julio 1998.

El endeudamiento para consumo se refiere a la serie "Préstamos al consumo" del Balance de las entidades financieras que publica el Banco Central de la República Argentina. Toma en cuenta los créditos para consumo acordados en pesos argentinos a personas físicas residentes en el país e incluye los préstamos otorgados bajo el sistema de tarjetas de crédito. La 
Juliana E. Vega / Karen Watkins

serie se deflactó utilizando el Índice de Precios al Consumidor del Gran Buenos Aires (IPC-GBA) con base abril 2008.

La medición del ingreso de las familias se aproxima por medio del Estimador Mensual de Actividad Económica (EMAE) que publica el Instituto Nacional de Estadística y Censos (INDEC). Es un indicador del curso de la actividad económica mensual que anticipa -de manera provisional- la variación del producto interno bruto trimestral. La actividad económica repercute en el ingreso de las familias a través de los factores de la producción (principalmente el trabajo) que se necesitan para la elaboración de bienes y servicios. La serie está desestacionalizada y se expresó como un número índice con base abril 2008.

Para cuantificar el costo de la financiación al consumo se utiliza la serie “Tasas de interés para préstamos personales", publicada por el BCRA. Los datos, divididos por plazos (hasta 180 días y más de 180 días), ponderan las tasas de préstamos personales y de tarjetas de crédito. Se generó una única serie con la media de ambas tasas y posteriormente se calculó la tasa de interés real conforme a la fórmula: [(1+ tasa de interés nominal del periodo)/ (1+ tasa de inflación del periodo)]-1, donde la tasa de inflación se obtuvo a partir del Índice de Precios al Consumidor del Gran Buenos Aires (IPC-GBA) con base abril 2008.

Como medida de desempleo se toman los datos de la tasa de desocupación que calcula el INDEC a partir de la Encuesta Permanente de Hogares (EPH), que se realiza trimestralmente. La tasa de desocupación se calcula como porcentaje entre la población desocupada y la población económicamente activa. Para generar una serie mensual se recurrió al proceso de desagregación temporal, mediante el método de Boot, Feibes y Lisman (1967) en primeras diferencias.

Para medir la confianza de las familias se emplea el ICC, elaborado por el Centro de Investigación en Finanzas de la Universidad Torcuato di Tella. El indicador mide la percepción de los individuos en tres áreas clave: el estado de la economía en general, la situación económica personal y las expectativas a mediano plazo.

\section{Identificación de los intervalos de crisis económicas en Argentina}

Con el fin de examinar la relación entre el volumen de préstamos al consumo y variables de ingreso, tasa de interés, desocupación y confianza del consumidor 
durante periodos normales y de crisis en Argentina, es importante comenzar con un análisis de identificación de los episodios de crisis económicas que tuvieron lugar en Argentina durante el periodo de estudio.

\subsection{Cambios interanuales en el PIB real}

El término crisis económica hace referencia a lo que técnicamente se conoce como recesión y que los economistas definen de manera sencilla como dos trimestres consecutivos con caídas en el PIB (Shiskin, 1974).

En la Figura 1 se presentan los cambios interanuales del PIB real trimestral; los puntos de la figura muestran las variaciones negativas de este indicador. Se puede validar que estas variaciones negativas coinciden con episodios de crisis conocidas: Crisis del tequila (1995-96), Crisis asiática/brasileña (199800), Crisis argentina (2001-02) y Crisis financiera internacional (2008-09). En el Cuadro 1 se presentan los periodos que corresponden a la definición de recesión antes expuesta.

\subsection{Pruebas con quiebre desconocido y conocido}

Se verifica la existencia de los periodos de crisis identificados anteriormente a través de pruebas de estabilidad estructural, tomando estos periodos, en primera instancia, como desconocidos. Para ello se corre el PIB (desestacionalizado) en función del tiempo y se comprueba la estabilidad de los parámetros mediante métodos de estimación recursiva. Las pruebas específicas que se aplicaron son las de residuos recursivos, el test CUSUM (que no dio significativo) y la prueba a un paso de pronóstico. Los periodos de crisis se vislumbran como los puntos donde se presentan cambios estructurales.

La misma lógica se sigue con las pruebas de quiebre conocido. Para robustez se someten a comprobación los puntos de quiebre obtenidos en el análisis anterior. Los procedimientos utilizados para puntos de quiebre conocidos son el test de Chow de cambio estructural y el test de predicción de Chow. En el Cuadro 2 se presentan los puntos de quiebre que dieron significativo; en números resaltados con negritas se destacan aquellos periodos identificados con al menos dos pruebas del conjunto de pruebas aplicadas, con lo cual se obtienen los periodos de crisis para Argentina de acuerdo a esta metodología. Éstos son los que se emplean en el estudio econométrico posterior. 
Juliana E. Vega / Karen Watkins

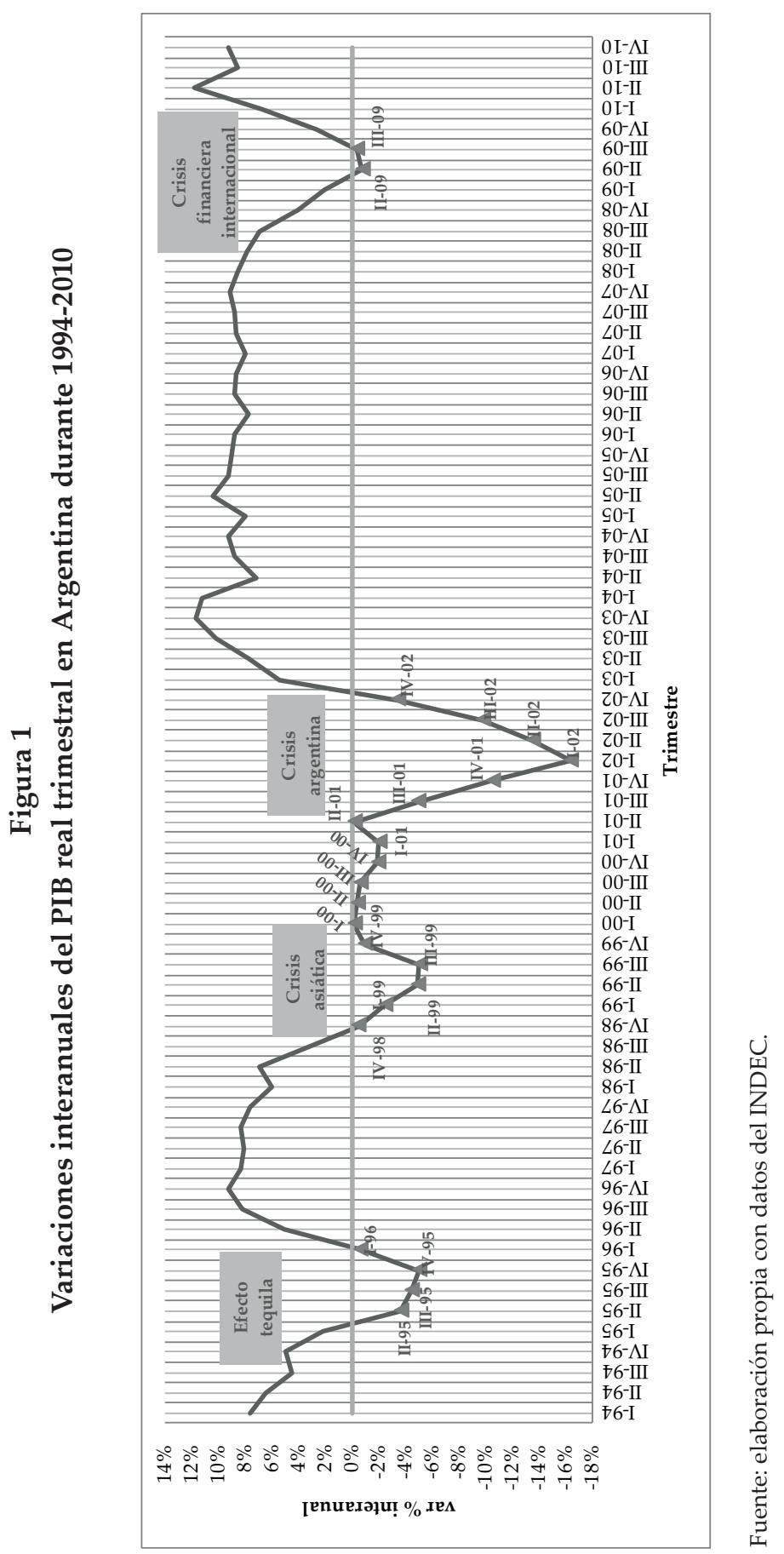




\section{Cuadro 1}

Periodos de crisis de acuerdo a variaciones interanuales del PIB real trimestral en Argentina

\begin{tabular}{lc}
\hline \multicolumn{1}{c}{ Crisis } & Periodo \\
\hline Efecto tequila & 1995:02-1996:01 \\
Crisis asiática & 1998:04-2001:02 \\
Crisis argentina & 2001:03-2002:04 \\
Crisis financiera internacional & 2009:02-2009:03 \\
\hline
\end{tabular}

Fuente: elaboración propia con datos del INDEC.

\section{Cuadro 2}

Identificación de periodos de crisis económicas en Argentina con pruebas de cambio estructural

\begin{tabular}{lcccc}
\hline & & \multicolumn{3}{c}{ Trimestres identificados } \\
\hline Crisis conocida & $\begin{array}{c}\text { Residuos } \\
\text { recursivos }\end{array}$ & One-step & $\begin{array}{c}\text { Chow } \\
\text { breakpoint }\end{array}$ & Chow forecast \\
Efecto tequila & $1995: 01-1995: 03$ & $1995: 01-1995: 03$ & & \\
Crisis asiática & & $1999: 02-2001: 02$ & 1999:02 - 2001:02 & \\
Crisis argentina & $2001: 03-2002: 03$ & $2001: 03-2002: 04$ & $2001: 03-2002: 04$ & \\
Crisis internacional & & $2008: 04-2009: 01$ & & 2008:04 - 2009:01 \\
\hline
\end{tabular}

Fuente: elaboración propia con datos del INDEC. 
Juliana E. Vega / Karen Watkins

\section{Análisis descriptivo}

En el siguiente conjunto de gráficos (figuras 2 a 6) se observa la evolución de cada una de las variables de estudio, distinguiendo con las áreas sombreadas los cuatro episodios de crisis detectados en el apartado anterior. En la primera gráfica se muestra la serie "Préstamos al consumo", donde destaca el despegue a partir del año 2003, justo después de la Crisis argentina. Esta tendencia al alza se vio levemente interrumpida durante la Crisis financiera internacional (2008-2009). Comparando con los demás tiempos de crisis, el de la Crisis argentina se caracteriza por tener el impacto negativo más profundo sobre el nivel de endeudamiento para consumo. Dentro de ese intervalo de crisis hay un pico en enero 2002, posiblemente relacionado con el término del régimen de convertibilidad que pesificó los créditos otorgados por el sistema financiero.

La evolución del EMAE (Figura 3) se caracteriza por una marcada estacionalidad con movimientos a la baja en los meses de enero de cada año y picos en mayo y diciembre. Al igual que con los préstamos al consumo, se recalca un periodo de crecimiento del EMAE a partir del año 2003. Por otro lado, se observan caídas en cada episodio de crisis, siendo la más pronunciada la de 20012002 (Crisis argentina).

La tasa de desocupación crece con cada periodo de crisis y luego cae otra vez en tiempos normales. La tendencia a la baja se agudiza a partir de la recuperación de la Crisis argentina en 2003, luego de experimentar los niveles más altos de desempleo del periodo de estudio con un máximo de $21.5 \%$ en junio de 2002 . El elevado desempleo se debió en parte a que, durante el periodo de convertibilidad, la sobreapreciación del peso deterioró la competitividad provocando una contracción en la producción nacional y consecuentemente en el empleo.

El comportamiento de la tasa de interés para préstamos personales (Figura 5) sigue un patrón ascendente durante los periodos de crisis, acentuándose más durante la Crisis argentina. Después de esta crisis, la tasa de interés para préstamos personales muestra una reducción estructural; aún con la Crisis financiera internacional, este indicador presenta niveles muy inferiores a los observados antes de 2003.

Las caídas en el nivel de confianza de los consumidores coinciden con los periodos de crisis, en particular con la Crisis argentina y la Crisis financiera internacional. El ICC presenta su nivel más bajo durante la Crisis argentina (28.4 en septiembre de 2002), para luego recuperarse hasta llegada la Crisis financiera internacional. Los niveles más altos de confianza se vislumbran entre estas dos crisis y posterior a la crisis internacional. 
Figura 2

Préstamos al consumo en Argentina durante 1994-2011

Préstamos personales

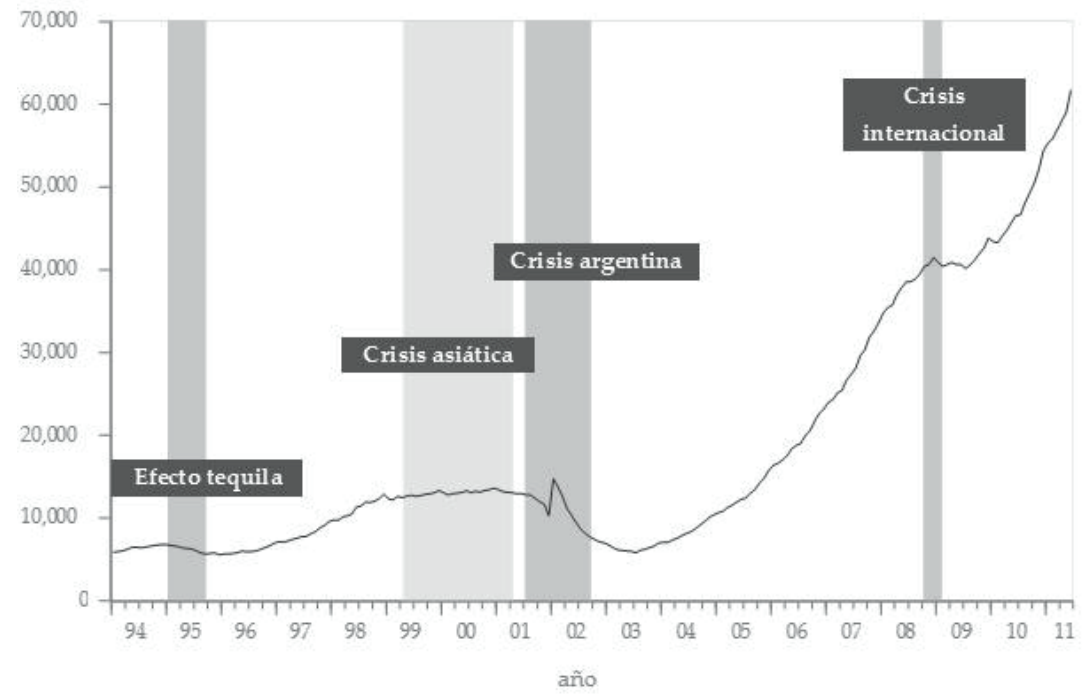

La serie se expresa en millones de pesos reales deflactada con el IPC-GBA, base abril 2008.

Fuente: elaboración propia con base en datos del BCRA.

\section{Figura 3}

\section{Estimador Mensual de Actividad Económica en Argentina durante 1994-2011}

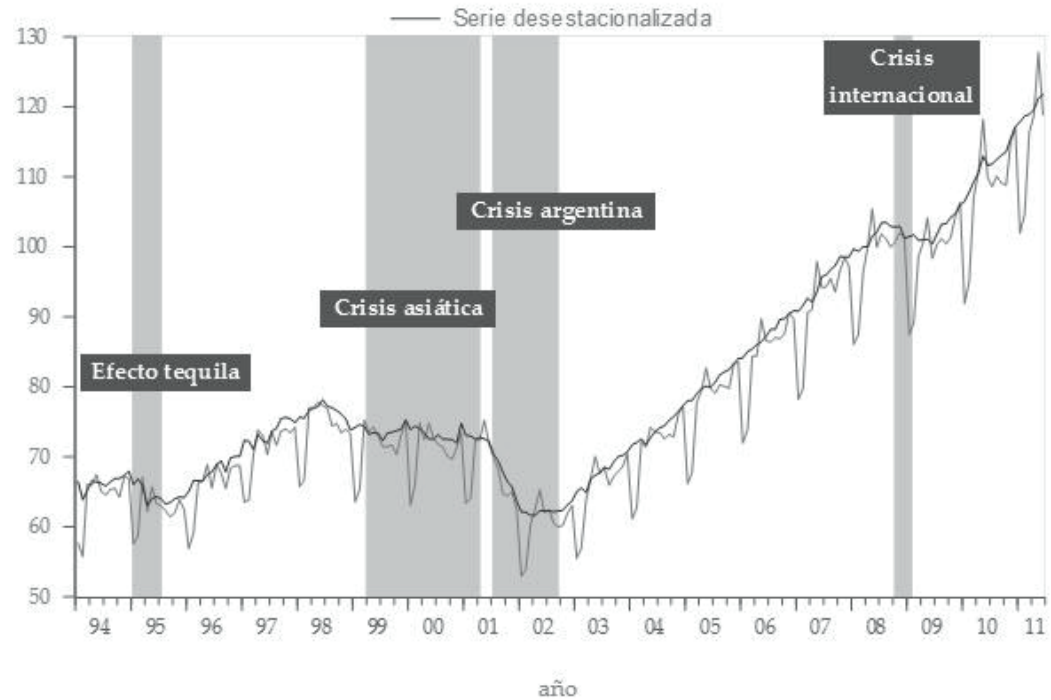

La serie se expresa como un número índice con base abril 2008.

Fuente: elaboración propia con base en datos del BCRA. 
Figura 4

Tasa de desocupación en Argentina durante 1994-2011

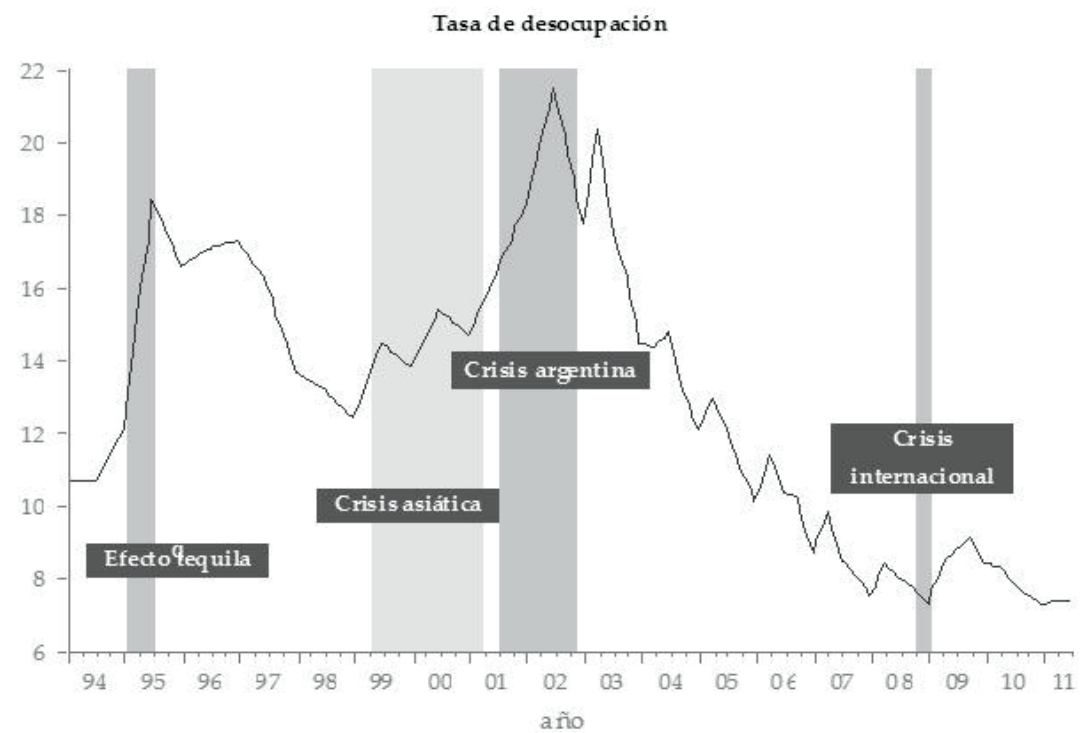

Fuente: elaboración propia con base en datos del INDEC.

\section{Figura 5}

Tasa de interés sobre préstamos personales en Argentina durante 1994-2011

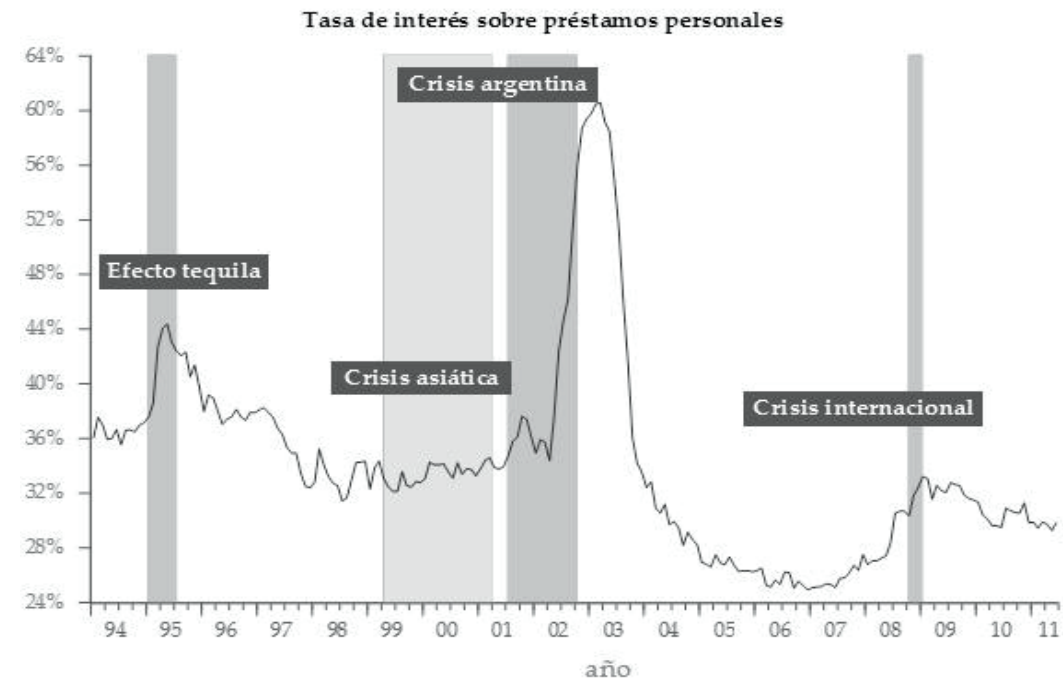

Serie promedio expresada en términos reales con base abril 2008.

Fuente: elaboración propia con base en datos del BCRA. 
Figura 6

Índice de confianza del consumidor en Argentina durante 1998-2011

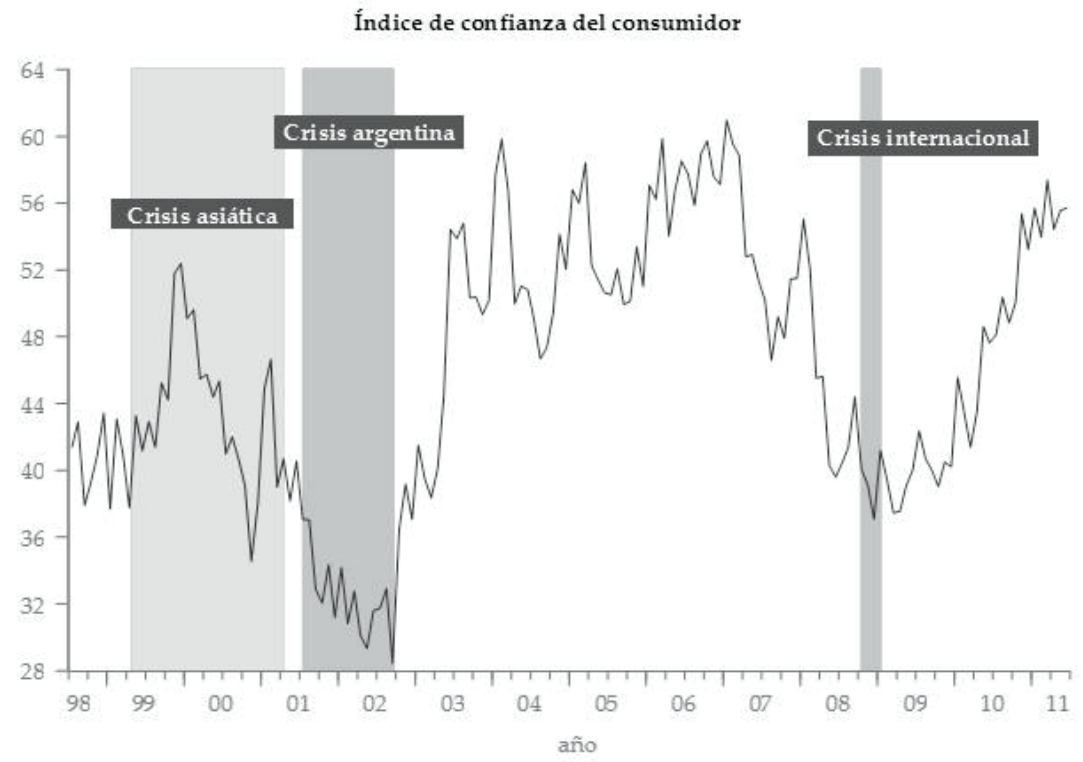

Fuente: elaboración propia con datos del Centro de Investigación en Finanzas, Universidad Torcuato di Tella.

\section{Análisis econométrico}

\subsection{Estimación general}

La relación entre los Préstamos al Consumo $(P C O N)$ y las variables Estimador Mensual de Actividad Económica (EMAE), Tasa de Desocupación (TD), Tasa de Interés para Préstamos Personales (TI) e Índice de Confianza del Consumidor (ICC) se describe mediante la siguiente ecuación:

$$
\operatorname{PCON}_{t}=\beta_{0}+\beta_{1} \mathrm{PCON}_{t-1}+\beta_{2} \mathrm{EMAE}_{t}+\beta_{3} T D_{t}+\beta_{4} T I_{t}+\beta_{5} \mathrm{ICC}_{t}+\mu_{t}
$$

Esta ecuación se corre primero para la muestra que comprende desde julio 1998 hasta junio 2011 (156 observaciones) y luego se excluye el ICC para abordar el periodo completo que abarca desde enero 1994 hasta junio 2011 (210 observaciones). Se toma en cuenta entre las variables explicativas la variable dependiente con rezago, para considerar el efecto dinámico que 
Juliana E. Vega / Karen Watkins

existe entre el volumen de préstamos al consumo pasado y presente. Antes de realizar las estimaciones, se remueve el componente estacional encontrado en todas las variables.

La ecuación se corre utilizando mínimos cuadrados ordinarios (OLS) con errores estándar consistentes en presencia de heteroscedasticidad (método de White). No es necesario corregir por autocorrelación, pues ésta no se observa de acuerdo a la prueba de Breusch-Godfrey. En cuanto a la multicolinealidad, la alta correlación entre las variables explicativas señala que este problema está latente, por lo que se transforman estas variables en primeras diferencias. Finalmente, debido a que no siempre las reacciones de los consumidores son instantáneas, se verifican otras especificaciones de la ecuación (1) que incluyen rezagos de las variables explicativas. Se consideran de uno hasta 12 rezagos para cada variable, de acuerdo a fuentes tales como The Conference Board, que mencionan que el crédito al consumo varía sólo después de que los ingresos han cambiado durante un año. Tinbergen (1942) encontró también que hay un rezago de doce meses entre el ingreso y el consumo.

En el Cuadro 3 se reportan los resultados generales obtenidos a partir de la ecuación (1), donde no se diferencia entre periodos normales y de crisis económicas.

Los resultados muestran que todas las variables contempladas se relacionan con los préstamos al consumo, tal como la teoría lo señala. Existen además rezagos en las decisiones de endeudamiento de las familias de acuerdo al comportamiento de las variables consideradas. El mayor rezago se observa en la tasa de desempleo, lo cual da indicios de que el desempleo inicialmente se considera transitorio y no es hasta varios meses después que afecta (de forma negativa) el endeudamiento para consumo. Por el contrario, el efecto de la tasa de interés sobre los préstamos al consumo es inmediato (y negativo). Tanto el ingreso de las familias, medido por el EMAE, como el ICC y el endeudamiento pasado impactan de forma positiva los préstamos al consumo. La relación directa entre ingreso y endeudamiento para consumo, así como el efecto del desempleo sobre esta deuda, dan evidencia a favor de la hipótesis del ingreso permanente.

\subsection{Estimación para periodos normales y de crisis económicas}

Con el fin de estudiar la relación entre las variables consideradas y los préstamos al consumo, diferenciando entre episodios normales y de crisis económicas en Argentina, se separa la ecuación (1) por periodos. Los periodos 
Crédito al consumo en Argentina durante Periodos normales y De CRISIS ECONÓmiCAS

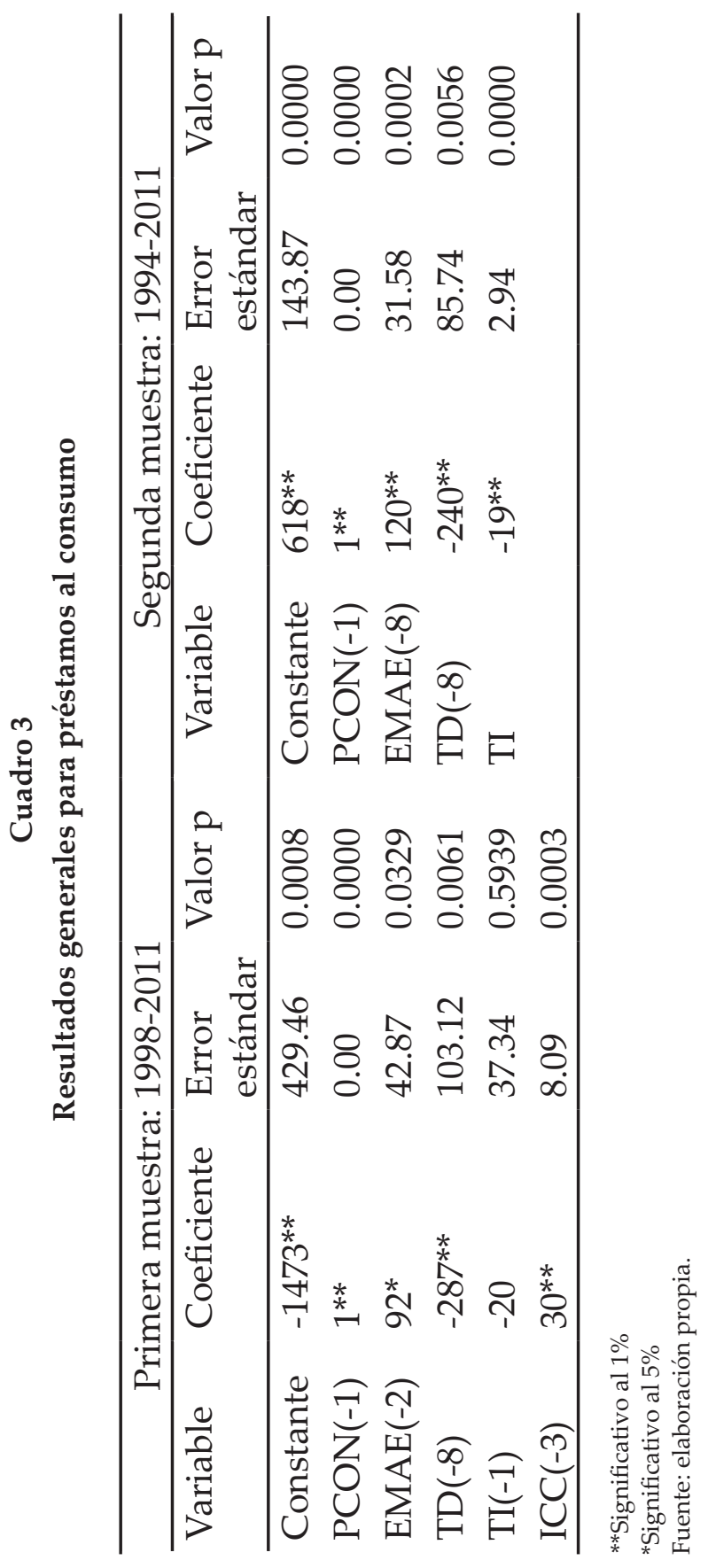


Juliana E. Vega / Karen Watkins

de crisis fueron identificados en la sección cinco del trabajo y se incorporan -ajustando los trimestres a meses- en la ecuación (2) como una variable dummy (0 para tiempos normales, 1 para tiempos de crisis económicas). Los periodos normales se añaden con otra variable dummy, que toma el valor de 1 para tiempos normales y 0 para episodios de crisis:

$$
\begin{aligned}
& \text { PCON }_{t}=\beta_{0}+\beta_{1} \text { PCON }_{t-1}{ }^{*} \text { CRISIS }+\beta_{2} \text { EMAE }_{t}{ }^{*} \text { CROSOS }+\beta_{3} \text { TD }_{t}{ }^{*} \text { CRISIS }+\beta_{4} \text { TI I }_{t}{ }^{*} \text { CRISIS }+\beta_{5} \text { ICC }_{t} \\
& { }^{*} \text { CRISIS }+\beta 6 \text { PCON }_{t-1}{ }^{*} \text { NOCRISIS }+\beta_{7} \text { EMAE }_{t}{ }^{*} \text { NOCRISIS }+\beta_{8} \text { TD }_{t}{ }^{*} \text { NOCRISIS }+\beta_{9} \text { TI }_{t} \\
& { }^{*} \text { NOCRISIS }+\beta_{10} \text { ICC }_{t}{ }^{*} \text { NOCRISIS }+\mu_{t}
\end{aligned}
$$

La ecuación (2) se corre utilizando la misma metodología que en la estimación de la ecuación (1), con excepción de la inclusión de rezagos, pues el fin es distinguir entre episodios normales y de crisis económicas. Los resultados se presentan en el Cuadro 4.

A partir de estos resultados se observa que los préstamos al consumo presentan inercia, ya que tanto para periodos normales como para episodios de crisis económicas, éstos se relacionan directamente con el endeudamiento del periodo anterior. El ICC es otra variable que muestra una correlación positiva con los préstamos al consumo, para los dos periodos de tiempo estudiados. Este hallazgo refleja la importancia de las expectativas de los consumidores sobre las decisiones de endeudamiento. De acuerdo a la prueba de Wald, el coeficiente del ICC en el periodo de crisis no es estadísticamente distinto al obtenido en el periodo de no crisis. Por otro lado, la tasa de desocupación tiene una correspondencia negativa con la deuda para consumo, la cual es significativa particularmente para tiempos normales. Posiblemente esto tenga lugar puesto que es en periodos de crisis económicas cuando el desempleo se vislumbra como temporal; en otros momentos éste puede impactar más fuertemente las decisiones de endeudamiento, por tratarse de una condición ajena a la situación económica nacional. Se observa también que en tiempos de crisis económicas la tasa de interés se mueve en dirección opuesta a los préstamos para consumo. A mayores tasas de interés menor es el endeudamiento, ya que por una parte éste resulta más caro y por otra, dada la crisis, las entidades financieras contraen los créditos. Sin embargo, la dirección de la relación entre la tasa de interés para préstamos personales y los préstamos al consumo no es concluyente para periodos normales. Finalmente, llama la atención que la correspondencia entre el EMAE y los préstamos al consumo no resulta significativa en ninguno de los dos periodos de estudio. 
CRÉdito al CONSUmo en Argentina duRANTE Periodos nORMALES y DE CRISIS ECONÓMICAS

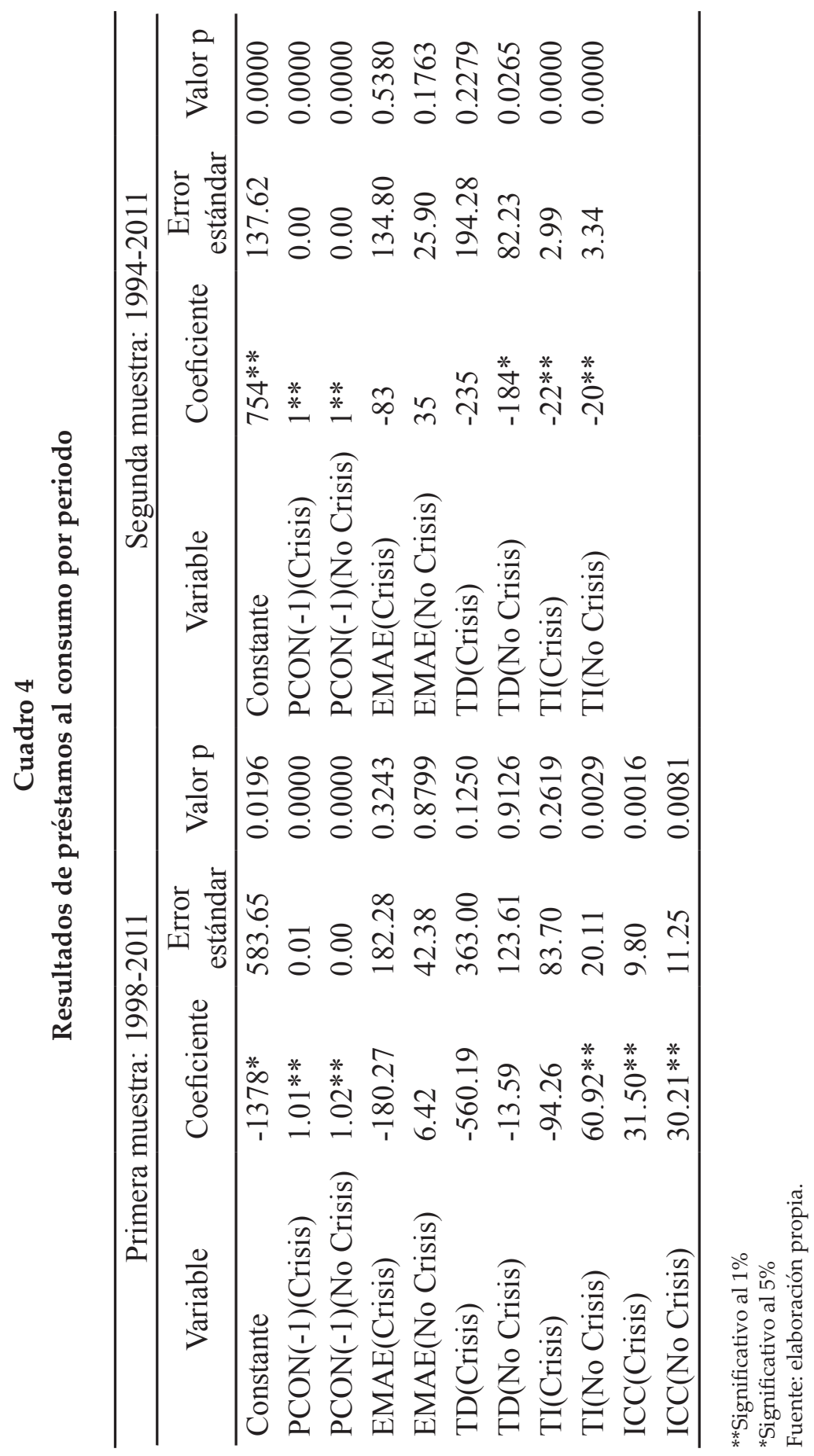


Juliana E. Vega / Karen Watkins

Se puede cuestionar que la tendencia en tiempos de crisis es que a medida que baja el ingreso aumentan los préstamos (para no sacrificar el consumo tras una situación temporal) y que en tiempos normales la disminución del ingreso se acompaña de un menor endeudamiento, posiblemente por tratarse estas caídas del EMAE como cambios estructurales permanentes. De ser así, nuevamente existe evidencia a favor de la hipótesis del ingreso permanente.

\section{Conclusiones}

La evolución de los préstamos al consumo en Argentina a lo largo de toda la línea de tiempo considerada, desde 1994 hasta 2011, muestra una clara tendencia hacia arriba. Algo que se puede deducir de esto es que la sociedad está revelando que prefiere consumir hoy antes que mañana. Esa modalidad de consumo se aprecia especialmente durante los últimos ocho años estudiados, posterior a la Crisis argentina. Pero, ¿se adapta también a otros aspectos propuestos por la teoría económica más allá de los gustos? $\mathrm{Al}$ intentar responder esto, los resultados de la ecuación general -la que no hace divisiones por contextos- da indicios que todo marcha conforme a la teoría. Esto es, los niveles más altos de endeudamiento para consumo están relacionados con mayores niveles de actividad económica, mayor confianza de los consumidores, menor desempleo y valores más bajos de las tasas de interés.

Conforme acercamos la lupa hacia lapsos particulares de tiempo (normales y de crisis económicas), es posible contar más detalles de la historia. Pareciera que los consumidores actúan de forma distinta si se ubican en una situación transitoria (crisis) a diferencia de un contexto normal (no crisis); por ejemplo, la tasa de desocupación tiene una correspondencia negativa y significativa con los préstamos al consumo sólo durante tiempos normales. Ya que es en periodos normales cuando el desempleo se vislumbra como permanente, éste afecta más fuertemente las decisiones de endeudamiento. También, hay sospechas de que cuando el ingreso cae en tiempos normales, se contraen los préstamos para consumo por considerarse seguramente una situación permanente. Por el contrario, la caída en el ingreso en épocas de crisis pareciera relacionarse de forma inversa con el endeudamiento, posiblemente para no sacrificar el consumo tras una situación temporal. Esto da evidencia a favor de la hipótesis del ingreso permanente. 
A futuro, se abren muchas interrogantes por responder, desde cuestiones de elección, de sustentabilidad y hasta efectos sobre el ciclo económico. Queda intentar mejorar la medición de las variables e incluir nuevas. Considerar no sólo desempleo, sino subocupación y condiciones del mercado laboral. Probar con medidas de costo financiero más amplias además de la tasa de interés. Añadir más estudios con variables psicológicas de percepción para intentar captar, por ejemplo, si los cambios en ingreso y desempleo son vistos como transitorios o permanentes. En definitiva, no renunciar a la tarea de entender y explicar el tema a fondo.

\section{Referencias}

Ashley, D. W. (2002), “The Demand for Consumer Credit”, Unpublished Master's Thesis, Virginia Polytechnic Institute and State University.

Barnes, S. y G. Young (2003), "The Rise in US Household Debt: Assessing its Causes and Sustainability", Bank of England Working Paper, 206.

Baumohl, B. (2007), The Secrets of Economic Indicators: Hidden Clues to Future Economic Trends and Investment Opportunities, 2a ed., Wharton School Publishing.

Boorstin, D. (2004), "Credit History: The Evolution of Consumer Credit in America", The Ledger: Economic Education Newsletter, 16.

Boot, J. C. G., W. Feibes y J. H. C. Lisman (1967), "Further Methods of Derivation of Quarterly Figures from Annual Data", Journal of the Royal Statistical Society, vol. 16, núm. 1, pp.65-75.

Calder, L. (2002), "The Evolution of Consumer Credit in the United States", en T. A. Durkin y M. E. Staten (Eds.), The Impact of Public Policy on Consumer Credit (pp. 336). Norwell, Massachusetts: Kluwer Academic Publishers.

Cox, R. (1948), The Economics of Instalment Buying, New York: The Ronald Press Company.

Chen, B. (2007), "An Empirical Comparison of Methods for Temporal Distribution and Interpolation at the National Accounts", BEA Working paper, 2007-03.

Chen Chen, K. y M. Chivakul (2008), “What Drives Household Borrowing and Credit Constraints? Evidence from Bosnia and Herzegovina", IMF Working Paper, 08/202.

Chow, G. C. (1960), "Tests of Equality Between Sets of Coefficients in Two Linear Regressions", Econometrica, vol. 28, núm. 3, pp. 591-603. 
Juliana E. Vega / Karen Watkins

Chrystal, K. A. y P. Mizen (2001), "Consumption, Money and Lending: A Joint Model for the UK Household Sector", Bank of England Working Paper, núm. 134.

Dauten, C. A. (1954), "A Fresh Approach to the Place of Consumer Credit in Economic and Financial Thinking", The Journal of Finance, vol. 9, núm. 2, pp. 111-123.

Deaton, A. (2005), "Franco Modigliani and the Life Cycle Theory of Consumption", Research Program in Development Studies and Center for Health and Wellbeing, 22.

Del Río, A. (2002), “El endeudamiento de los hogares españoles: evolución y factores explicativos", Boletín Económico Banco de España, 11(228):4754.

Desroches, B. y M. A. Gosselin (2002), "The Usefulness of Consumer Confidence Indexes" in the United States, Bank of Canada Working Paper, 02-22.

Dion, D. P. (2006), “Does Consumer Confidence Forecast Household Spending?", MPRA Paper, núm. 902, University Library of Munich, Germany.

Duesenberry, J. S. (1949), Income, Saving, and the Theory of Economic Behavior, Harvard University Press.

Durkin, T. A. y M. E. Staten (2002), "Introduction", en T. A. Durkin y M. E. Staten (Eds.), The Impact of Public Policy on Consumer Credit, Kluwer Academic Publishers, pp. 336.

Earl, P. E. y S. Kemp (2002), The Elgar Companion to Consumer Research and Economic Psychology, Edward Elgar Publishing.

Enthoven, A. (1957), "The Growth of Instalment Credit and the Future of Prosperity", American Economic Review, vol. 47, núm. 6, pp. 913-929.

Friedman, M. (1957), A Theory of the Consumption Function, National Bureau of Economic Research.

Gross, D. B. y N. S. Souleles (2001), "Do Liquidity Constraints and Interest Rates Matter for Consumer Behavior? Evidence from Credit Card Data", The Quarterly Journal of Economics, vol. 117, núm. 1, pp. 149-185.

Haberler, G. (1942), Consumer Instalment Credit and Economic Fluctuations, New York: National Bureau of Economic Research.

Jiménez, F. (2001), Macroeconomía: Enfoques y Modelos, Tomo I, Lima: Fondo Ed. PUCP.

Keynes, J. M. (1936), The General Theory of Employment, Interest, and Mone, London, Macmillan (reimpreso en 2007).

Kisselgoff, A. (1952), Factors Affecting the Demand for Consumer Instalment Sales Credit, vol. 1, New York: National Bureau of Economic Research. 
Lewis, R. E. (1956), "Some Factors in the Growth of Consumer Credit", The Journal of Finance, vol. 11, núm. 2, pp. 249-256.

Logemann, J. (2008), “Different Paths to Mass Consumption: Consumer Credit in the United States and West Germany during the 1950s and 60s", Journal of Social History, pp. 525-559.

Ludvigson, S. C. (2004), "Consumer Confidence and Consumer Spending", Journal of Economic Perspectives, vol. 18, núm. 2, pp. 29-50.

Magri, S. (2002), "Italian Households' Debt: Determinants of Demand and Supply", Temi di discussione del Servizio Studi no. 454, Banca d'Italia.

Makin, J. H. (1977), Macroeconomía, (V. M. Armer, Trans. 1. ed.), México, D.F., Nueva Editorial Interamericana, S.A. de C.V.

Mason, R. (2000), "The Social Significance of Consumption: James Duesenberry's Contribution to Consumer Theory", Journal of Economic Issues, vol. 34, núm. 3, pp. 553-572.

Morales R., L. y O. A. Yáñez (2006), “Créditos de consumo bancarios: evolución reciente 1997-2005", Serie Técnica de Estudios, núm. 003. Superintendencia de Bancos e Instituciones Financieras (SBIF)(003).

Neagu, F. y A. Margarit (2007), “Risks to Romanian Financial Stability Stemming from the Household Sector", IFC Bulletin, núm. 26, pp. 75-108.

Palley, T. (2007), "The Relative Income Theory of Consumption: A Synthetic Keynes-Duesenberry-Friedman Model", Working Paper Series, núm. 170, Political Economy Research Institute.

Park, S. (1993), "The Determinants of Consumer Installment Credit", Federal Reserve Bank of St. Louis Review, November/December, pp. 23-38.

Pearce, D. K. (1985), "Rising Household Debt in Perspective”, Economic Review, Federal Reserve Bank of Kansas City, pp. 3-17.

Shiskin, J. (1974), "The Changing Business Cycle", The New York Times, p. 222.

Soto, C. (2004), “Desempleo y consumo en Chile”, Documento de Trabajo, núm. 258, Banco Central de Chile.

Strebkov, D. (2005), "Household Borrowing Behavior in Russia", Problems of Economic Transition, vol. 48, núm. 5, pp. 22-48.

"The Conference Board", <http://www.conference-board.org/>.

Tinbergen, J. (1942), “Does Consumption Lag Behind Incomes?", The Review of Economics and Statistics, vol. 24, núm. 1, pp. 1-8.

Tudela, M. y G. Young (2005), "The Determinants of Household Debt and Balance Sheets in the United Kingdom", Bank of England Working Paper Series, núm. 266. 
Juliana E. Vega / Karen Watkins

Weller, C. E. (2007), “Need or Want: What Explains the Run-up in Consumer Debt?, Journal of Economic Issues, vol. 41, núm. 2, pp. 583-591.

Wilcox, J. A. (2007), "Forecasting Components of Consumption with Components of Consumer Sentiment", Working paper, University of California, Berkeley. 